LAWRENCE LIVERMORE N A T IO N A L LABORATORY

Simulations of rapid

pressure-induced solidification in molten metals

M. V. Patel, F. H. Streitz

October 15, 2003

APS Shock 2003

Portland, OR, United States

July 20, 2003 through July 25, 2003 
This document was prepared as an account of work sponsored by an agency of the United States Government. Neither the United States Government nor the University of California nor any of their employees, makes any warranty, express or implied, or assumes any legal liability or responsibility for the accuracy, completeness, or usefulness of any information, apparatus, product, or process disclosed, or represents that its use would not infringe privately owned rights. Reference herein to any specific commercial product, process, or service by trade name, trademark, manufacturer, or otherwise, does not necessarily constitute or imply its endorsement, recommendation, or favoring by the United States Government or the University of California. The views and opinions of authors expressed herein do not necessarily state or reflect those of the United States Government or the University of California, and shall not be used for advertising or product endorsement purposes. 
UCRL-PROC-200298

\title{
SIMULATIONS OF RAPID PRESSURE-INDUCED SOLIDIFICATION IN MOLTEN METALS
}

\author{
Mehul V. Patel* and Frederick H. Streitz* \\ ${ }^{*}$ Lawrence Livermore National Laboratory, L-045, 7000 East Ave, Livermore CA 94550
}

\begin{abstract}
The process of interest in this study is the solidification of a molten metal subjected to rapid pressurization. Most details about solidification occurring when the liquid-solid coexistence line is suddenly transversed along the pressure axis remain unknown. We present preliminary results from an ongoing study of this process for both simple models of metals $(\mathrm{Cu})$ and more sophisticated material models (MGPT potentials for Ta). Atomistic (molecular dynamics) simulations are used to extract details such as the time and length scales that govern these processes. Starting with relatively simple potential models, we demonstrate how molecular dynamics can be used to study solidification. Local and global order parameters that aid in characterizing the phase have been identified, and the dependence of the solidification time on the phase space distance between the final $(\mathrm{P}, \mathrm{T})$ state and the coexistence line has been characterized.
\end{abstract}

\section{INTRODUCTION}

The nature of the solidification process has long been an area of scientific and technical interest. The confluence of peaked experimental interest in this problem and recent theoretical and computational advances that may allow for a breakthrough in understanding, have resulted in many recent studies [1]. While solidification is most commonly studied by varying temperature at fixed (ambient) pressure, i.e. freezing, the goal of this research is to study the other axis, namely rapid, pressure-induced solidification. The questions to be answered are numerous and include the determination of the final state (crystalline or glassy?), the transition path, and the time scales for the relevant processes. Atomistic simulations show great potential for answering many of the detailed questions describing how a disorder to order phase transition proceeds.

Figure 1 shows the phase diagram of tantalum, with the horizontal arrow depicting the isothermal compression path being studied in this work. This path lies close to an isentrope, a path being investigated in the experiments that are also part of this effort [9]. (Also shown is the principle hugoniot, which is the locus of all possible states attainable by a shock

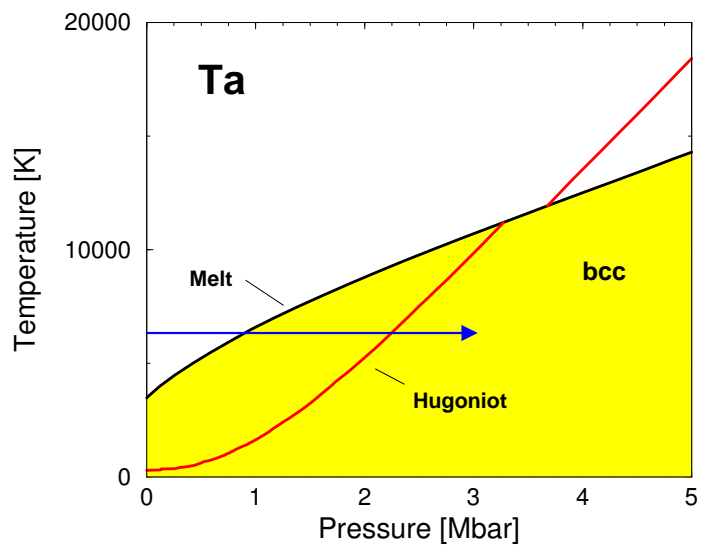

FIGURE 1. Phase diagram of tantalum. The arrow demonstrates an isothermal compression, which is the solidification path being studied. Also shown is the principle hugoniot.

from ambient temperature and pressure). In this paper, we will present some preliminary results studying the dependence of solidification time on the degree of over-pressurization. This is analogous to the time-temperature-transition studies that look at the dependence of the solidification rate on the degree of 


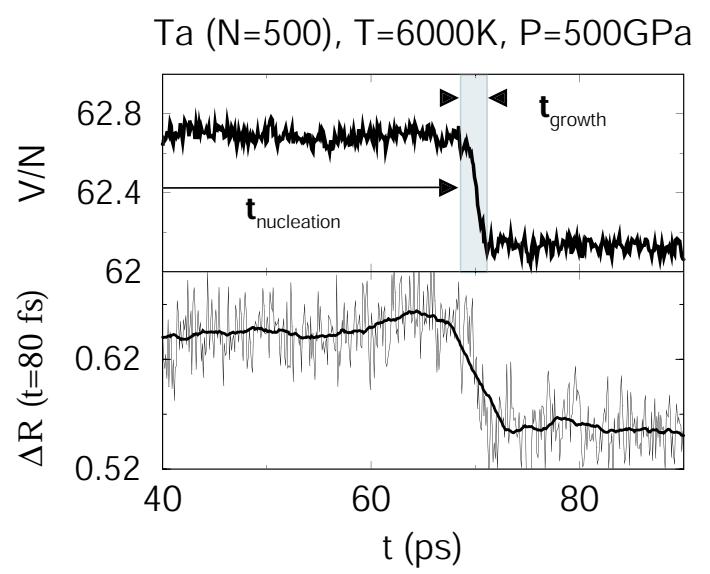

FIGURE 2. Typical simulation output for Ta at 6000 $\mathrm{K}$, showing phase transition signatures in the volume and average displacement. The nucleation time and growth time are noted on the volume trace.

under-cooling.

\section{METHODOLOGY}

Constant NPT molecular dynamics simulations using a stochastic Langevin thermostat are employed for this study. Copper is modeled using the EAM potential developed by Johnson and Oh [2]. For Ta, we utilize the more sophisticated Model Generalized Pseudopotential Theory (MGPT) potentials [4]. These potentials were developed for metals having more complex electronic structure, and have been used with success in a number of other high pressure studies [5].

An equilibrated molten sample is prepared and subjected to a sudden compression, reaching final pressure in 1-2 ps. After pressurization, the system is observed until a phase transition, if any, occurs. Evidence for the transition can be seen in several global observables such as the energy, density (atomic volume), diffusion constant, and pair correlation function. Local order parameters [6] were also used to study the transition and are especially useful when a metastable phase is present.

For example, Figure 2 shows output from a typical tantalum simulation. One can see signatures of the transition in the atomic volume as well as the average displacement (in this case plotted for an interval of

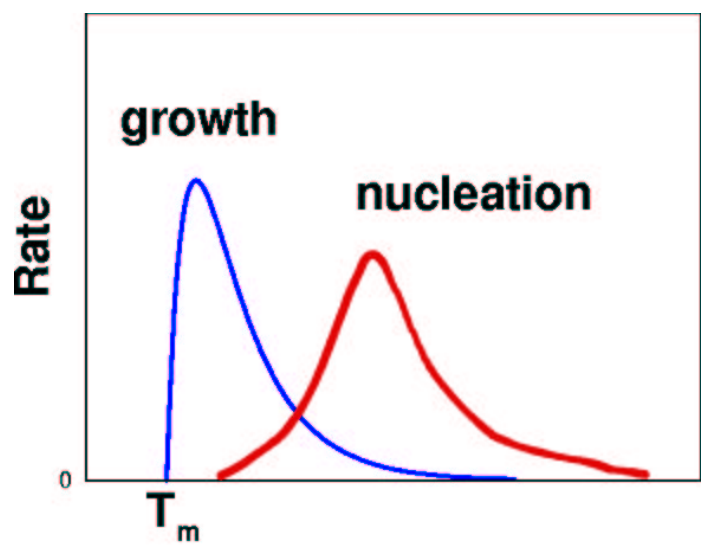

FIGURE 3. Sketch showing the dependence of the nucleation and growth rates on the degree of under-cooling below the melting temperature, $T_{m}$. The solidification time is minimized in the region where the nucleation rate and growth rate overlap.

$80 \mathrm{fs})$. Figure 2 also shows how we define the solidification timescales: $t_{\text {solidification }}=t_{\text {nucleation }}+t_{\text {growth }}$.

Using our machine-precision restart capability (even for this stochastic, parallel code), we can study the simulation starting from just before the transition using a variety of analysis tools. With so many parameters to contend with (final state, loading rates, etc), we choose to focus on final pressure (analagous to under-cooling) as the independent variable and fix the others.

\section{TIME-PRESSURE-TRANSITION ANALYSIS FOR EAM COPPER}

\section{TTT Plots}

In classical nucleation theory (developed to describe the freezing process), nucleation and growth rates are described by Arrhenius equations that depend on quantities such as the nucleation energy and the diffusive energy barrier. They have been sketched in Figure 3 which highlights the non-monotonic dependence of the nucleation and growth rates on the amount of under-cooling. The overlap region in which both nucleation and growth are favored defines the domain of the Time-Temperature-Transition plots, in which the transition time is plotted against 


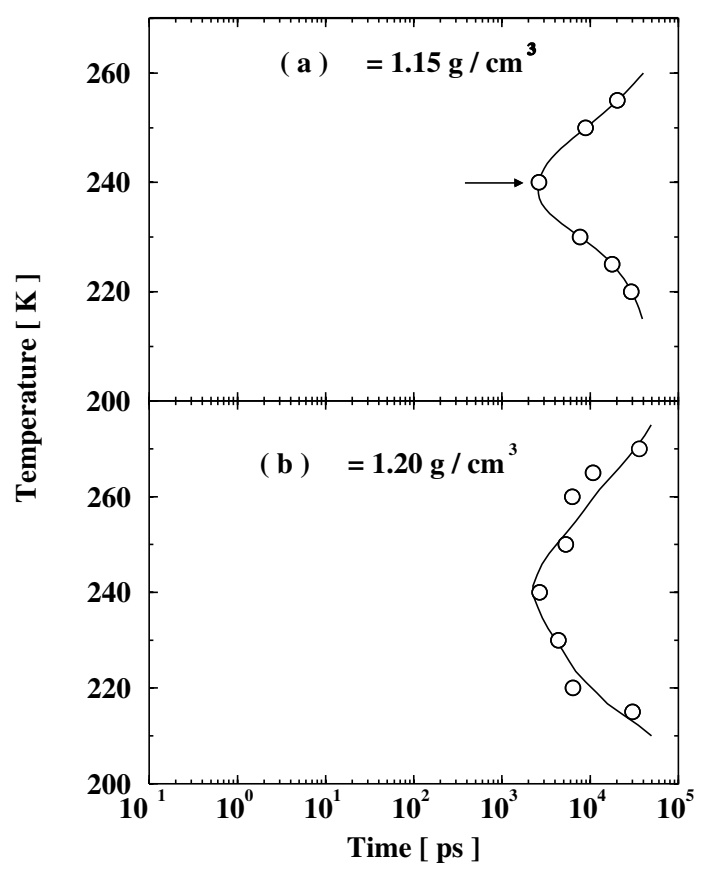

FIGURE 4. An example of a Time-TemperatureTransition (TTT) plot obtained from MD simulations. Average crystallization time (open circles) for water as a function of temperature shown for two densities (a) $1.15 \mathrm{~g} / \mathrm{cm}^{3}$ and (b) $1.20 \mathrm{~g} / \mathrm{cm}^{3}$. Plot adapted from Ref. [7].

the final temperature as shown in Figure 4.

The intersection of the nucleation rate and growth rate in Figure 3 defines the optimum under-cooling temperature (corresponding to the "nose" in Figure 4) where the transition time is minimized. As is clear from the figure, changing the final temperature by only $10 \%$ can lead to an order of magnitude change in the crystallization time. Given the limited time scales accessible to MD simulation, such an order of magnitude shift in expected crystallization time can be the difference between observing a transition during the simulation or not. In the present study, we seek to determine if similar behavior is present along the pressure axis, namely, is there an optimal degree of over-pressurization that minimizes the liquid-solid transition time?

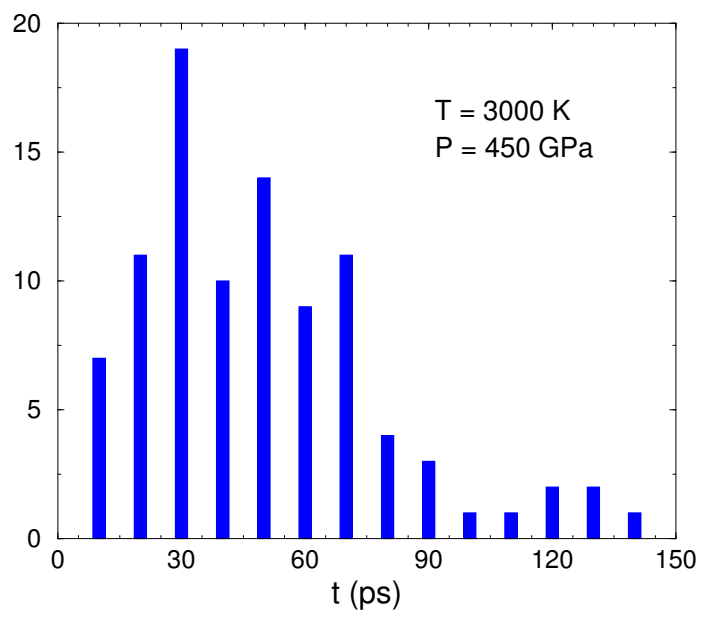

FIGURE 5. Distribution of solidification times in copper $(\mathrm{N}=500$ atoms) for a fixed final temperature and pressure.

\section{Preliminary Results}

The solidification times, as defined in Figure 2, were obtained for a variety of different final pressures and temperatures for a large set of independent samples having $\mathrm{N}=500$ atoms. We obtain an average solidification time with error estimates for each temperature and final pressure by analyzing the (rather broad) distribution of independent solidification times. Figure 5 displays one such distribution. We plot the resulting average solidification time against the final pressure to create a timepressure-transition (TPT) plot, shown in in Figure 6 for $\mathrm{EAM}$ copper at $\mathrm{T}=3500 \mathrm{~K}$. The transition time initially decreases with increasing over-pressure, but eventually reaches a plateau, which defines a minimum solidification time. The initial decrease is expected, since the transition time must diverge to infinity at the melt line. However, the lack of a transition time minimum stands in stark contrast to the behaviour demonstrated in a TTT plot, where there is a clear minimum in the transition time as a function of under-cooling. The viscosity is a sharply increasing function of pressure, which should serve to hinder the nucleation process and drive the solidification times up. The lack of a clear minimum may indicate that the relative loss of mobility with overpressure is compensated by the increased thermodynamic driving force for solidification, and suggests that the formation of a metastable state by rapid over- 


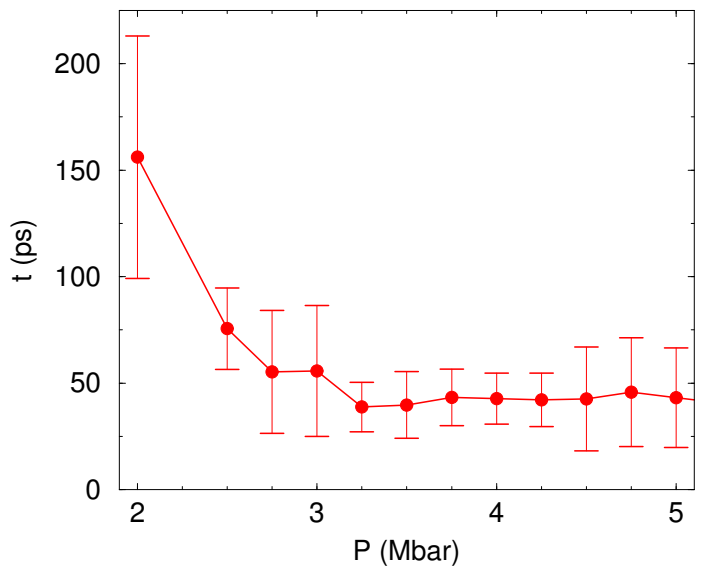

FIGURE 6. Time-Pressure-Transition (TPT) plot for EAM copper at $\mathrm{T}=3500 \mathrm{~K}(\mathrm{~N}=500)$ showing the dependence of the solidification time on the final pressure.

pressurization is unlikely. A detailed discussion of these issues will be presented elsewhere [8].

Similar behavior is observed in TPT plots over a range of different temperatures. If we define the optimal pressure to be that pressure at which the transition time plateaus to its minimum value, we can plot these optimal phase space points on the phase diagram as is shown in Figure 7. At the level of the error bars shown, these points seem to follow the melt curve. Further analysis using larger simulation cells is needed to make better quantitative comparisons and to determine the role of finite size effects in these preliminary calculations.

\section{CONCLUSION}

Atomistic simulations of pressure induced solidification pose many challenges but also show potential for answering basic questions about this process, and for bridging the gap to the next length and time scales. Some preliminary results have been presented here to illustrate the kinds of questions that we hope to answer. Simulations on larger systems are underway to assess the magnitude of finite size effects and to study any size scaling that can be used advantageously.

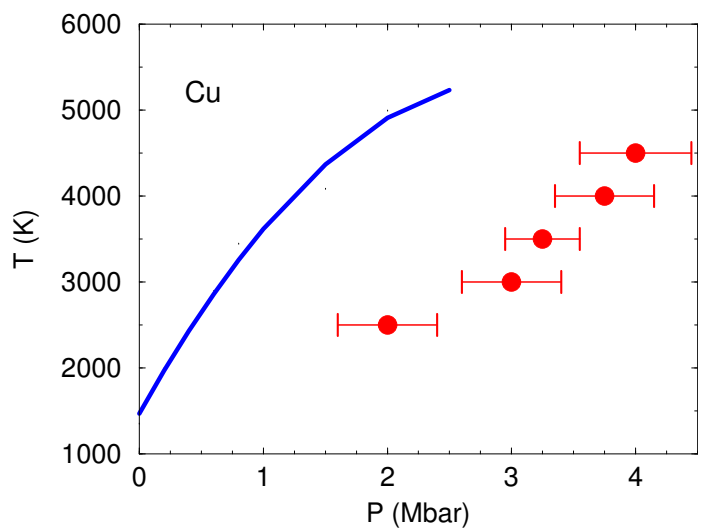

FIGURE 7. Locus of phase space points where solidification appears optimal plotted along side the copper melt line [3].

\section{ACKNOWLEDGMENTS}

Work performed under the auspices of the U.S. DOE at the University of California/Lawrence Livermore National Laboratory under contract W-7405-ENG48.

\section{REFERENCES}

1. Day, C., Physics Today, 7, 24 (2003).

2. Oh, D. J., and Johnson, R. A., J. Mater. Res., 3, 471 (1988).

3. Jeong, J., and Chang, K. J., J. Phys. Cond. Matt., 11, 3799 (1999).

4. Yang, L. H., Söderlind, P., and Moriarty, J. A., Phil. Mag. A, 81, 1355 (2001).

5. Moriarty, J., Belak, J., Rudd, R., Söderlind, P., Streitz, F., and Yang, L., J. Phys. Cond. Matt., 14, 2825 (2002).

6. Steinhardt, P., Nelson, D. R., and Ronchetti, M., Phys. Rev. B, 28, 784 (1983).

7. Yamada, M., Mossa, S., Stanley, H. E., and Sciortino, F., Phys. Rev. Lett., 88, 35501 (2002).

8. Patel, M.V. and Streitz, F.H., in preparation (2003).

9. Nguyen, J.H., Orlikowski, D., Streitz, F.H., Minich, R., Holmes, N.C., and Moriarty, J.A., in preparation (2003). 